\title{
Does Removal of Subchondral Cortical Bone Provide Sufficient Resection Depth for Treatment of Cam Femoroacetabular Impingement?
}

\author{
Penny R. Atkins BS, Stephen K. Aoki MD, Ross T. Whitaker PhD, \\ Jeffrey A. Weiss PhD, Christopher L. Peters MD, Andrew E. Anderson PhD
}

Received: 25 October 2016/Accepted: 16 March 2017/Published online: 24 March 2017

(C) The Association of Bone and Joint Surgeons (R 2017

\begin{abstract}
Background Residual impingement resulting from insufficient resection of bone during the index femoroplasty is the most-common reason for revision surgery in patients with cam-type femoroacetabular impingement (FAI). Development of surgical resection guidelines therefore could reduce the number of patients with persistent pain and reduced ROM after femoroplasty.
\end{abstract}

The institution of one or more of the authors (PRA, SKA, RTW, JAW, CLP, AEA) has received, during the study period, funding from the National Institutes of Health (R01-EB016701, P41-GM103545, R01-GM083925, R21-AR063844), and the LS-Peery Discovery Program in Musculoskeletal Research.

One of the authors certifies that he (SKA), or a member of his immediate family, has or may receive payments or benefits, during the study period, an amount of USD 10,000-USD 100,000, from Stryker Medical (Kalamazoo, MI, USA).

One of the authors certifies that he (CLP), or a member of his immediate family, has or may receive payments or benefits, during the study period, an amount of USD 100,001-USD 1,000,000, from Zimmer Biomet (Warsaw, IN, USA), and an amount of less than USD 10,000, from Conextions Medical (Salt Lake City, UT, USA). All ICMJE Conflict of Interest Forms for authors and Clinical Orthopaedics and Related Research ${ }^{\circledR}$ editors and board members are on file with the publication and can be viewed on request.

Clinical Orthopaedics and Related Research ${ }^{\mathbb{R}}$ neither advocates nor endorses the use of any treatment, drug, or device. Readers are encouraged to always seek additional information, including FDAapproval status, of any drug or device prior to clinical use.

Each author certifies that his or her institution approved the human protocol for this investigation, that all investigations were conducted in conformity with ethical principles of research, and that informed consent for participation in the study was obtained.

P. R. Atkins, S. K. Aoki, J. A. Weiss, C. L. Peters,

A. E. Anderson $(\bowtie)$

Department of Orthopaedics, University of Utah, 590 Wakara

Way, Room A100, Salt Lake City, UT 84108, USA

e-mail: Andrew.Anderson@hsc.utah.edu
Questions/purposes We asked whether removal of subchondral cortical bone in the region of the lesion in patients with cam FAI could restore femoral anatomy to that of screened control subjects. To evaluate this, we analyzed shape models between: (1) native cam and screened control femurs to observe the location of the cam lesion and establish baseline shape differences between groups, and (2) cam femurs with simulated resections and screened

P. R. Atkins, R. T. Whitaker, J. A. Weiss, C. L. Peters,

A. E. Anderson

Department of Bioengineering, University of Utah, Salt Lake

City, UT, USA

R. T. Whitaker, J. A. Weiss, A. E. Anderson

Scientific Computing and Imaging Institute, University of Utah, Salt Lake City, UT, USA

R. T. Whitaker, J. A. Weiss

School of Computing, University of Utah, Salt Lake City, UT, USA

\section{A. E. Anderson}

Department of Physical Therapy, University of Utah, Salt Lake City, UT, USA 
control femurs to evaluate the sufficiency of subchondral cortical bone thickness to guide resection depth.

Methods Three-dimensional (3-D) reconstructions of the inner and outer cortical bone boundaries of the proximal femur were generated by segmenting CT images from 45 control subjects ( 29 males; 15 living subjects, 30 cadavers) with normal radiographic findings and 28 nonconsecutive patients (26 males) with a diagnosis of cam FAI based on radiographic measurements and clinical examinations. Correspondence particles were placed on each femur and statistical shape modeling (SSM) was used to create mean shapes for each cohort. The geometric difference between the mean shape of the patients with cam FAI and that of the screened controls was used to define a consistent region representing the cam lesion. Subchondral cortical bone in this region was removed from the 3-D reconstructions of each cam femur to create a simulated resection. SSM was repeated to determine if the resection produced femoral anatomy that better resembled that of control subjects. Correspondence particle locations were used to generate mean femur shapes and evaluate shape differences using principal component analysis.

Results In the region of the cam lesion, the median distance between the mean native cam and control femurs was $1.8 \mathrm{~mm}$ (range, $1.0-2.7 \mathrm{~mm}$ ). This difference was reduced to $0.2 \mathrm{~mm}$ (range, -0.2 to $0.9 \mathrm{~mm}$ ) after resection, with some areas of overresection anteriorly and underresection superiorly. In the region of resection for each subject, the distance from each correspondence particle to the mean control shape was greater for the cam femurs than the screened control femurs (1.8 mm, [range, $1.1-2.9 \mathrm{~mm}$ ] and $0.0 \mathrm{~mm}$ [range, $-0.2-0.1$ $\mathrm{mm}$ ], respectively; $\mathrm{p}<0.031$ ). After resection, the distance was not different between the resected cam and control femurs $(0.3 \mathrm{~mm}$; range, $-0.2-1.0 ; \mathrm{p}>0.473)$.

Conclusions Removal of subchondral cortical bone in the region of resection reduced the deviation between the mean resected cam and control femurs to within a millimeter, which resulted in no difference in shape between patients with cam FAI and control subjects. Collectively, our results support the use of the subchondral cortical-cancellous bone margin as a visual intraoperative guide to limit resection depth in the correction of cam FAI.

Clinical Relevance Use of the subchondral cortical-cancellous bone boundary may provide a method to guide the depth of resection during arthroscopic surgery, which can be observed intraoperatively without advanced tooling, or imaging.

\section{Introduction}

One challenging aspect of hip arthroscopy is properly contouring the lesion in patients with cam femoroacetabular impingement (FAI) [25]. One of the most-common reasons for revision arthroscopy is underresection (68\%-90\% of revision arthroscopy procedures) [2, 23]. Although less common, overresection also has been noted as a cause of iatrogenic femoral neck fracture, loss of the normal joint suction seal, or loss of congruency $(0.05 \%-1.9 \%$ of arthroscopies) $[9,10,30]$. Careful assessment of the resection during surgery may minimize complications. Intraoperatively, fluoroscopy attempts to recreate clinical radiographic views that show the area of the cam lesion before, during, and after resection [4, 16, 17, 27]. Arthroscopic views provide qualitative assessment, including confirmation that ROM is improved by the resection $[21,27]$. However, there is no standard approach to evaluate arthroscopic images to determine the extent to which the resection has normalized femoral anatomy.

Experimental and computational techniques have been used to develop resection depth guidelines [18, 19, 24, 29]. For example, an experimental cadaver study showed that resections less than $30 \%$ of femoral head-neck diameter were safe in terms of avoiding fracture [19]. The primary limitation to that study and similar research in this area is that guidelines were based on results from cadaveric femurs from a normal population or generalized models of anatomy [24, 29]. A more-recent study used an ovine model of cam FAI and found that resections of up to $9 \mathrm{~mm}$ reduced the failure load by nearly $20 \%$, but even the bones that underwent the largest resections failed at loads higher than those expected during daily activities [18]. Bone density and cortical thickness are elevated in the region of the cam lesion in patients with FAI, which suggests that the femoral neck in cam FAI femurs is stronger compared with that in control subjects $[1,26]$.

We have found that the margin between subchondral cortical bone and underlying cancellous bone, as observed radiographically and arthroscopically, provides a straightforward method to guide the depth of the resection during femoroplasty (Fig. 1). However, the extent to which a resection based on this boundary improves proximal femoral anatomy in patients with cam FAI has not been quantified. A major impediment to evaluation of the efficacy of this or any resection guideline is the difficulty in quantifying the baseline anatomic shape of the normal hip. Statistical shape modeling (SSM) offers the ability to objectively assess true, three-dimensional (3-D) anatomic variation across a population or between selected groups $[3,11]$. By analyzing 3-D reconstructions generated from volumetric medical images, SSM reduces the bias and subjectivity that may occur when quantifying femoral anatomy using radiographs or single image slices of volumetric data.

Using 3-D CT reconstructions and SSM, we asked whether removal of subchondral cortical bone in the region 


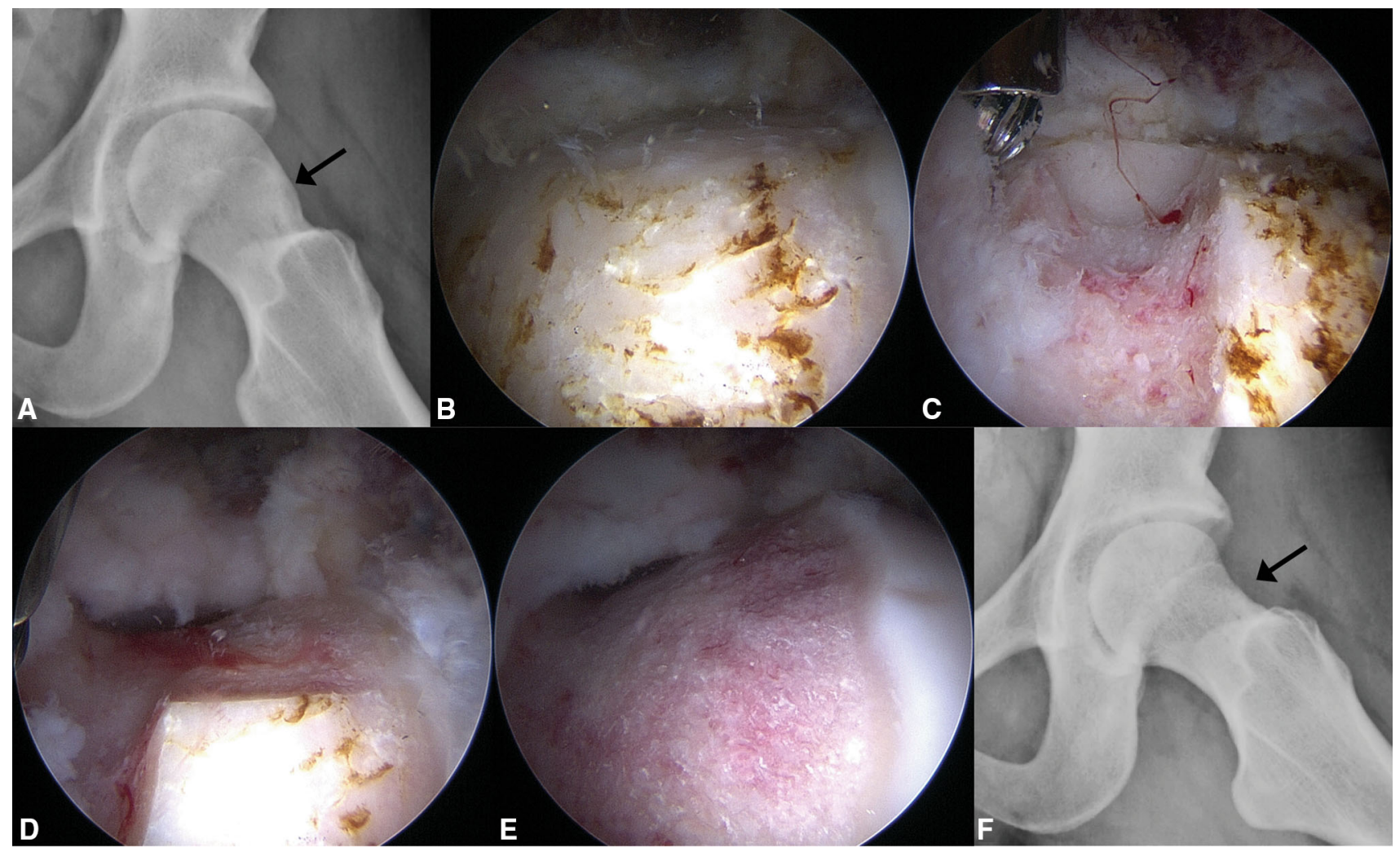

Fig. 1A-F A female patient with cam FAI was treated with femoroplasty using the sclerotic subchondral cortical bone thickness as a guide for resection depth. (A) Her preoperative frog-leg lateral radiograph shows the sclerotic region of the cam lesion (arrow). Intraoperative arthroscopic views from a $70^{\circ}$ scope through the anterolateral portal show (B) the sclerotic region, (C) the initial resection trough where the depth was based on the sclerotic subchondral cortical bone thickness, (D) continuation of the resection based on trough depth and patient anatomy, and (E) the completed resection. (F) The patient's postoperative frog-leg lateral radiograph shows where the sclerotic region has been removed (arrow).

studies between 2005 and 2012; living subjects and cadavers were considered for the control cohort. Patients with cam FAI $(n=28)$ represented a convenience sample of nonconsecutive patients recruited solely for research purposes between February 2005 and January 2009 (n = 15) and January 2011 and January 2012 ( $\mathrm{n}=13$ ). All patients had positive radiographic and clinical examination findings, including restricted ROM and pain elicited by the impingement examination, as assessed by an orthopaedic surgeon (CLP) with more than 15 years of experience treating FAI. Living subjects and cadavers were recruited for the control group to increase sample size. Living controls $(n=20)$ were recruited via word-of-mouth for a study conducted between April 2008 and July 2010; cadavers (n = 59) had undergone previous imaging for basic science studies. All potential controls (ie, living subjects and cadavers) were screened for radiographic evidence of cam FAI, using a digitally reconstructed radiograph of the frogleg lateral position generated from CT images (see below) [12]. The $\alpha$ angle was measured by a member of the study team with 10 years of medical imaging experience (AEA). 
Table 1. Summary of subject cohort demographics presented as median (range)

\begin{tabular}{lllll}
\hline Cohort & Male/female & Age $($ years $)$ & Weight $(\mathrm{kg})$ & BMI $\left(\mathrm{kg} / \mathrm{m}^{2}\right)$ \\
\hline Patients with cam FAI $(\mathrm{n}=28)$ & $26 / 2$ & $23(16-47)$ & $81(52-107)$ & $25(19-33)$ \\
Screened controls $(\mathrm{n}=45)$ & $29 / 16$ & $28(15-55)$ & $81(49-117)$ & $24(16-39)$ \\
$\mathrm{p}$ Value & & 0.152 & 0.496 & 0.901 \\
\hline
\end{tabular}

FAI femoroacetabular impingement.

Femurs with an $\alpha$ angle greater than $60^{\circ}$ were excluded $(\mathrm{n}=34)$ [5], leaving 45 screened controls $(\mathrm{n}=15$ living subjects, $\mathrm{n}=30$ cadavers). Demographics (ie, age, weight, and BMI) did not differ between patients with cam FAI and controls (Table 1).

CT images of the proximal femur of all subjects were acquired using a SOMATOM Definition ${ }^{\circledR} 128$ CT scanner (Siemens AG, Munich, Germany) (15 control subjects, 15 patients with cam FAI), HiSpeed ${ }^{\mathbb{R}}$ CTi Single Slice Helical CT scanner (GE Healthcare, Chicago, IL, USA) (30 control cadaver femurs), or LightSpeed ${ }^{\mathbb{R}}$ VCT $^{\circledR}$ scanner (GE Healthcare) (13 patients with cam FAI). Images were acquired at 100 to $120 \mathrm{kVp}, 512 \times 512$ acquisition matrix, 0.625 to $1.0 \mathrm{~mm}$ slice thickness, 0.9 to 1.0 pitch, and 100 to $200 \mathrm{mAs}$ with variable fields of view. Three-dimensional CT images were used to segment and reconstruct surfaces of the inner and outer cortex of the proximal femur (Fig. 2A) [1, 11].

The cam lesion was identified by first performing shape analysis between the native cam and control femurs (Fig. 2B), as described previously [1]. Correspondence particles, which serve as the basis to determine shape variation, were placed on the outer femoral cortex of the 28 cam FAI and 45 control femurs. Next, mean shapes were generated to represent the proximal femur of native cam and control subject populations. These mean shapes served as the basis for identification of the region of resection, specifically the distance between the mean surfaces was calculated to identify the difference in outer topology of the mean femurs between the native cam and control populations (Fig. 2C, left). The region of simulated resection then was defined as the region of the femoral head-neck junction where the outer topology of the mean native cam femur varied from that of the mean control femur by greater than $1 \mathrm{~mm}$ (Fig. 2C). Use of this 1-mm threshold on the mean cam shape provided a region that was thought to be an appropriate representation of the cam lesion, as assessed by a hip arthroscopist with 9 years of experience treating FAI (SKA). This region covered the femoral head-neck junction well, but limited the resection such that it did not extend past the junction between the neck and greater trochanter. To simulate a resection on each femur surface, the region identified on the mean cam shape was then mapped back onto each of the cam femur surfaces. In this region, the surface of the outer cortex was projected onto the surface of the inner cortex in MATLAB ${ }^{\circledR}$ Version 7.10 (MathWorks, Natick, MA, USA) (Fig. 2D, right). The simulated resections were smoothed locally to remove edge effects using 3-matic Version 10.0 (Materialise, Leuven, Belgium).

The extent (ie, areal coverage) of the resection was described using a set of angles from the center of the femoral head relative to each of the three anatomic axes. This was accomplished by mapping the areal coverage of the region of resection onto each of the three anatomic planes of the femur in MATLAB ${ }^{\circledR}$. Resection volume and surface area then were quantified in 3-matic.

SSM for the analysis of shape variation after the simulated resection followed a similar protocol as that described previously for native femurs [1]. First, correspondence particles were placed for the entire population, including the native cam, resected cam, and control femurs (Fig. 2E), and used to generate mean shapes for each of these groups. From this analysis, the difference in shape between the mean native cam and resected cam shapes and the mean control shape was measured by calculating the distance between surfaces. Although SSM used input shapes from three separate groups, only two groups were evaluated simultaneously such that either native cam or resected cam populations were compared with the control population (Fig. 2F). To ensure the optimization of correspondence particles across shapes and consistent interpretation of shape variation, the native cam, resected cam, and screened control femurs were all included in this second analysis.

The spatial locations of correspondence particles can be used to describe the shape of each femur and the shape variation across a population, overall and specific to any region of interest. In the region of resection of each subject, the spatial location of each correspondence particle was compared with that of the same correspondence particle on the mean control shape. The distances were evaluated for each correspondence particle to identify shape variability over the region between cohorts.

Principal component analysis (PCA) was conducted on the correspondence particle locations from SSM to determine shape variation associated with cam FAI and to evaluate the sufficiency of resection. PCA provides a method to reduce high-dimensional data (ie, 
Fig. 2A-F The flowchart is shown for the computational protocol, which used statistical shape modeling (SSM) to objectively evaluate whether resection of the subchondral bone of the cam lesion restored anatomy to that of a screened control population. (A) Three-dimensional (3-D) models of the inner and outer cortex were created from CT images. (B) Correspondence particles were placed on the 3-D surfaces of each subject. (C) The morphologic difference in the mean cam and mean control shape was identified by SSM; this difference established the region where subchondral bone should be removed. (D) This region then was applied to 3-D models of each patient with cam femoroacetabular impingement (FAI) to generate a simulated resection. (E) SSM was again applied to control subject femurs, native cam femurs, and resected cam femurs. (F) Shape variation was determined through comparison of correspondence particles and mean shapes of the native cam and resected cam femurs with the control femurs.

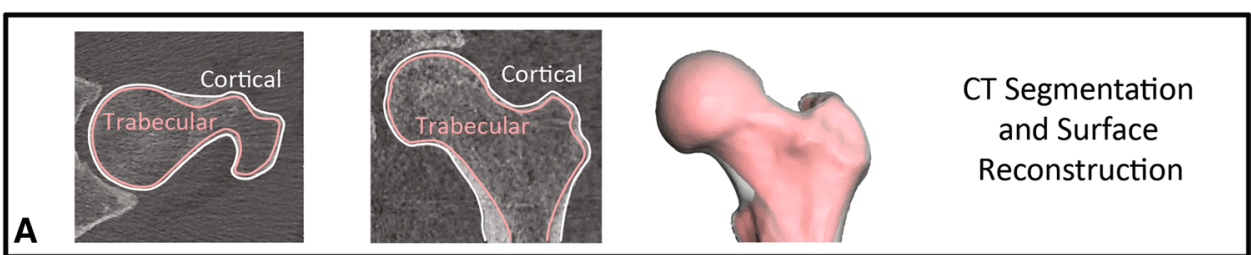

A
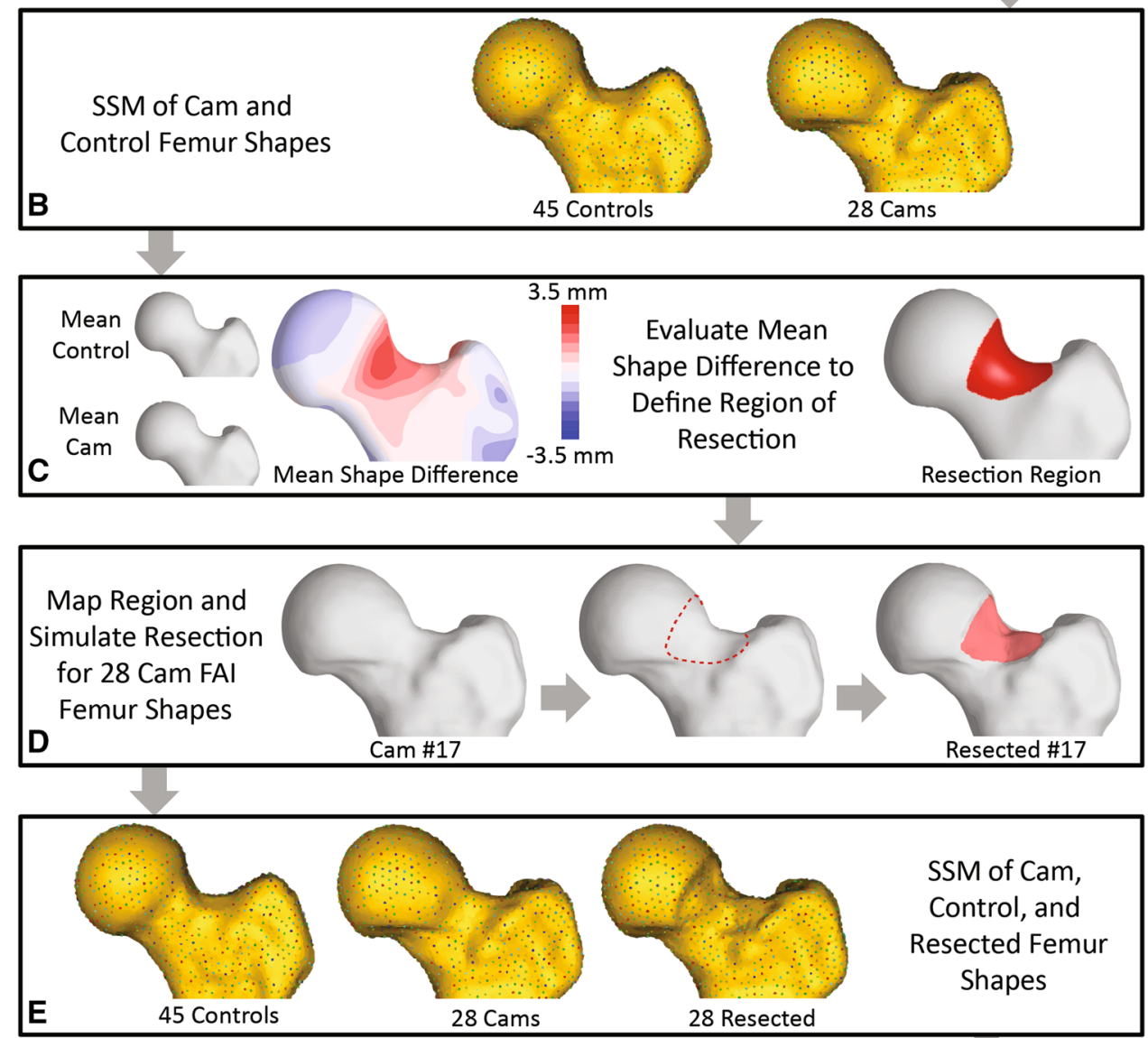

Evaluate Differences in

(1) Native Cam

Versus Control

(2) Resected Cam Versus Control correspondence particle locations) into fewer modes of variation, which best describe the variance in the data set. The results of PCA indicate modes of shape variation equal to the number of input shapes, with the first mode capturing the largest variance and each subsequent mode capturing less of the population variance than the previous mode. From PCA, each shape was represented by loading values for each of the modes describing shape variation (eg, similar to how a cylinder can be described using values of height and radius). Herein, PCA loading values were statistically evaluated to determine modes of variation which represented distinct shape variation between the native cam or resected cam and control groups.
Subject demographics, descriptive data regarding the resection (ie, depth and size), and distances between subject and mean correspondence particles in the region of resection were evaluated for normality using Shapiro's rank-sum test. Group differences of these metrics were evaluated using a Wilcoxon rank-sum test or Student's ttest based on the results of the normality evaluation. The nonspurious modes of variation from PCA were determined by analysis against random noise using a technique termed parallel analysis [8]. From these, overall group differences were evaluated using Hotelling's T-squared test [14], which is a multivariate generalization of Student's ttest that provides an overall analysis of group differences 
from the PCA loading values. Student's t-test then was used to determine the modes of variation that included distinct shape variations between groups. Significance was set at a probability less than 0.05 for all tests and Finner's method was used to control for multiple comparisons [7]. It was not possible to evaluate differences between mean shapes using statistical tests, as a single mean shape represents each cohort. Accordingly, the surface distance between the two mean shapes (ie, native cam to control, resected cam to control) was quantified and plotted using a color map. For consistency, all data were presented as median (range). All statistical analyses were completed using R@ Version 3.1.2 (R Foundation for Statistical Computing, Vienna, Austria) [22].

\section{Results}

\section{Morphometrics of Native Cam Femurs}

Before resection, the mean group shape for the native cam femur was 1.8 (range, 1.0-2.7) mm larger (Fig. 3) in the region identified for simulated resection (Fig. 2C). This region extended from $-1^{\circ}$ to $70^{\circ}$ from lateral to anterolateral in the axial plane (Fig. $4 \mathrm{~A}$ ), $57^{\circ}$ to $149^{\circ}$ from superolateral to inferolateral in the coronal plane (Fig. 4B), and $-1^{\circ}$ to $136^{\circ}$ from superior to anteroinferior in the sagittal plane (Fig. 4C) on the mean cam shape. In addition to the shape variation over the head-neck junction, the shape difference between the mean native cam and mean control femurs showed variation over the entire proximal femur (Fig. 3). Qualitatively, the medial border of the femoral head did not extend as far medially in patients with

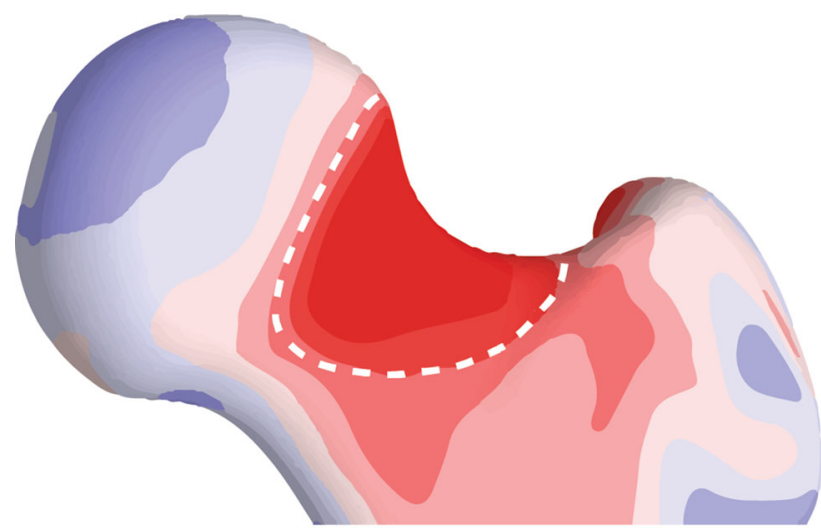

$-2 \mathrm{~mm}$

$2 \mathrm{~mm}$

Fig. 3 An anterior view is shown of the quantitative comparison between the mean control shape and the mean native cam shape. The surface distance was mapped on the mean control shape. The shape variation over the surface to be resected, outlined with a dashed white line, had a maximum deviation between shapes of $2.7 \mathrm{~mm}$. cam FAI and the shape of the proximal greater trochanter had more curvature medially in the axial plane, which is consistent with a previous analysis [11].

The first 10 PCA modes included $87 \%$ of the overall shape variation and were found to be nonspurious from parallel analysis. Together, the loading values from these 10 modes identified differences in overall shape between the native cam and control populations as determined from Hotelling's T-squared test $(\mathrm{p}<0.001)$. Of these 10 modes, four (Modes 1, 5, 6, and 8) described distinct shape differences between the two populations as identified by the results of the Student's t-test comparing loading values between groups $(\mathrm{p}=0.024,0.021,0.023$, and $<0.001$, respectively) (Fig. 5). These modes accounted for 31.3\%, $4.8 \%, 3.9 \%$, and $2.4 \%$ of the overall shape variation, respectively. Qualitatively, Mode 1 described overall variation in AP and mediolateral widths, Modes 5 and 6 described variations in the anterosuperior head-neck junction and height and curvature changes of the greater trochanter, and Mode 8 described variations of the posterior greater trochanter and mediolateral width of the femoral head.

In the region of the identified cam lesion of each subject, the distance between each correspondence particle and the same mean control shape correspondence particle was greater for the cohort of native cam femurs than for the cohort of control femurs of the mean distance for each correspondence particle (median, $1.8 \mathrm{~mm}$ [range, 1.1-2.9

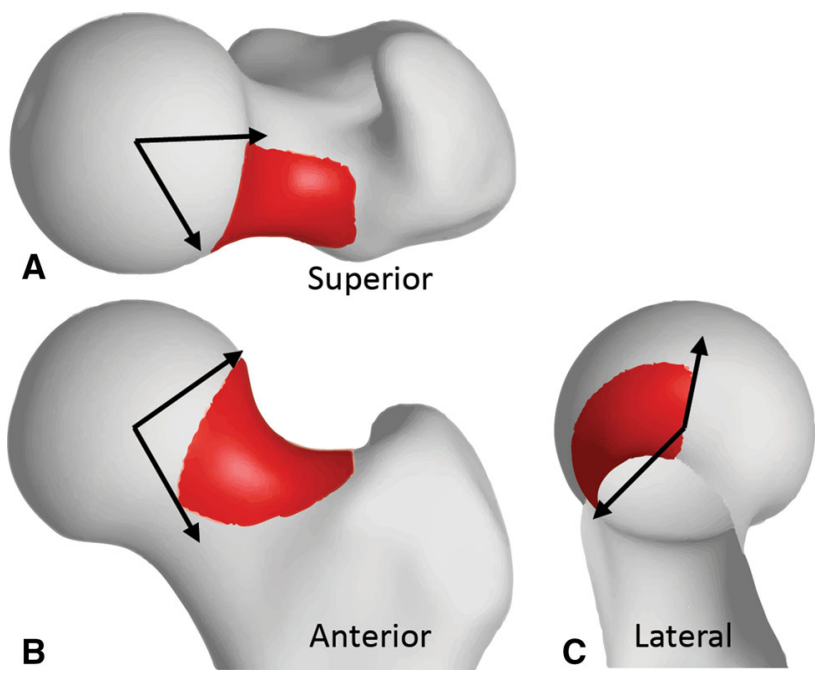

Fig. 4A-C The region of resection shown on the mean native cam shape was determined based on overall shape variation between patients with cam FAI and screened control subjects. The region was located primarily in the anterolateral head-neck junction, spanning $71^{\circ}, 93^{\circ}$, and $138^{\circ}$ in the (A) axial, (B) coronal, and (C) sagittal planes, respectively. The angle was measured clockwise from lateral in the superior view and from superior in the anterior and lateral views. The greater trochanter has been removed in the sagittal view to better observe the extent of the resection. 


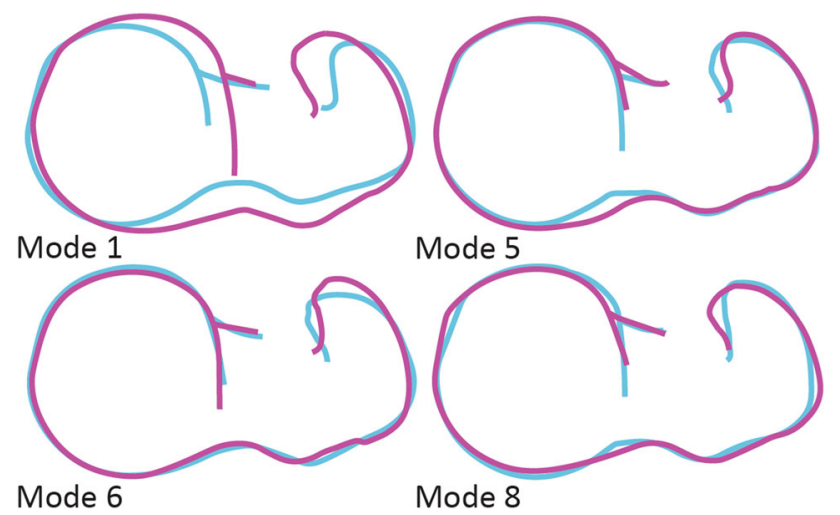

Fig. 5 Outlines of the two-dimensional projections of the femur surfaces from a superior view represent the variation associated with plus (magenta) and minus (blue) two SDs of the four principal component analysis modes which represent shape differences between the native cam and control populations.

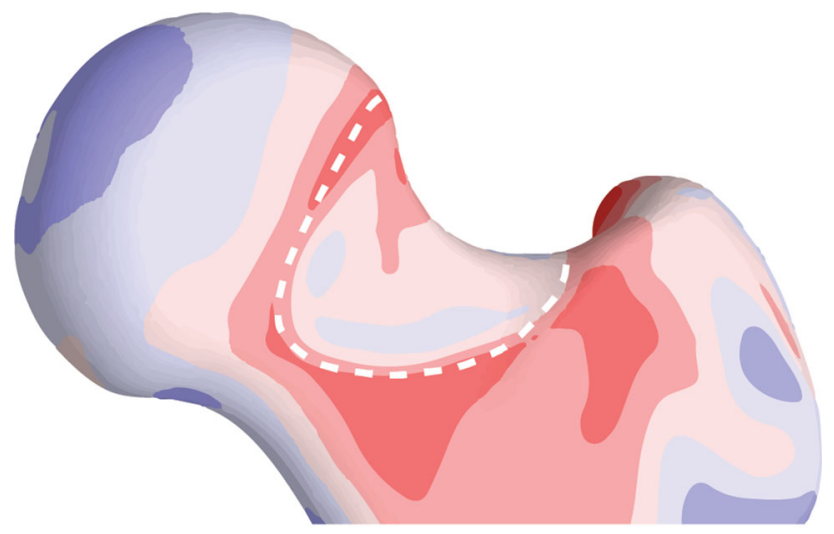

$-2 \mathrm{~mm}$

$2 \mathrm{~mm}$

Fig. 6 An anterior view is shown of the quantitative comparison between the mean control shape and the resected cam shape. The surface distance was mapped on the mean control shape. The shape variation over the surface of resection, outlined with a dashed white line, showed $0.9 \mathrm{~mm}$ underresection superiorly and $0.2 \mathrm{~mm}$ overresection anteriorly.

$\mathrm{mm}$ ] versus $0.0 \mathrm{~mm}$ [range, -0.2 to $0.1 \mathrm{~mm}$ ], $\mathrm{p}<0.031$ ). Although, the difference in correspondence particle distances was expected, it provided statistical evidence that there was a lesion present in the hips with symptomatic cam FAI.

\section{Morphometrics of Resected Cam Femurs}

For the simulated resection on the cohort of cam femurs, removal of subchondral cortical bone in the region of the cam lesion resulted in maximum resection depths with a median of $3.5 \mathrm{~mm}$ (range, 1.8-7.7 $\mathrm{mm}$ ), resected volumes of $1552 \mathrm{~mm}^{3}$ (range, $881-3348 \mathrm{~mm}^{3}$ ), and resected surface areas of $1034 \mathrm{~mm}^{2}$ (range, 686-1246 mm²). After resection, the difference between the mean resected cam shape and the mean control shape was reduced to $0.2 \mathrm{~mm}$ (range, $-0.2-0.9 \mathrm{~mm}$ ) in the region of resection. Analysis of the mean resected cam and control shapes indicated the maximum overresection occurred anteriorly and the maximum underresection superiorly (Fig. 6).

The first 10 PCA modes, which included $87 \%$ of the overall shape variation, were found to be nonspurious and together identified differences in overall shape between the resected cam and control populations $(\mathrm{p}<0.001)$. Of these 10 modes, only one (Mode 8) described a distinct shape difference between the two populations $(p=0.004)$ and accounted for $2.3 \%$ of the overall shape variation. The variation captured in Mode 8 was similar to Mode 8 described between the native cam and control femurs (Fig. 5).

In the region of the resection, distances to the set of mean control correspondence particle locations were not different between the resected cam femurs and the control femurs (median, $0.3 \mathrm{~mm}$ [range, $-0.2-1.0 \mathrm{~mm}$ ] versus 0.0 $\mathrm{mm}$ [range, -0.2 to $0.1 \mathrm{~mm}$ ], $\mathrm{p}>0.473$ ). This lack of difference indicated that resection of subchondral cortical bone resulted in anatomy similar to that of control subjects in the region of resection.

\section{Discussion}

In the treatment of cam FAI, overresection of a cam lesion may predispose the hip to femoral neck fracture or loss of the normal hip suction seal, while underresection is associated with persistent impingement-related symptoms. Assessment of proper resection depth intraoperatively can be challenging, especially for the inexperienced hip arthroscopist. We theorized that removal of subchondral cortical bone alone in the region of the cam lesion would yield femoral anatomy that was not different from control femurs. To evaluate this, we analyzed shape models between: (1) native cam and screened control femurs to observe the location of the cam lesion and establish baseline shape differences between groups, and (2) cam femurs with simulated resections and screened control femurs to evaluate the sufficiency of subchondral cortical bone thickness to guide resection depth. We found that removal of subchondral cortical bone reduced the deviation between resected cam and control femurs to less than a millimeter in the region of resection. In addition, the shape variation was eliminated over the region of the resection, as evidenced by the reduction in distance between the correspondence particles of the resected cam femurs and the mean control femur. Finally, PCA indicated that the number of modes representing distinct shape differences between groups was reduced from four to one after resection. Thus, collectively, 
our results support the use of the subchondral corticalcancellous bone margin as a visual, intraoperative guide for resection depth in the correction of cam FAI. Fortunately, this boundary provides real-time feedback as it is clearly visible without additional operative tooling or imaging (Fig. 1). However, the cortical-cancellous boundary only provides a guide to limit resection depth; the surgeon still must identify the areal extent of the cam lesion based on his or her clinical knowledge and expertise.

Our study does have some limitations. First, our definition of the region of resection was based on a 1-mm threshold on the shape difference between mean shape of native cam and control femurs. We implemented this approach to aid in the automatic definition of a resection region for each patient with cam FAI, which reduced subjectivity and bias in this regard. However, the 1-mm threshold used to outline the simulated resection was based only on qualitative inspection of what was deemed to be an appropriate resection boundary on the mean cam femur shape. Use of this standardized region may have misidentified the anterosuperior location or over- or underestimated the true areal coverage of the cam lesion on a subjectspecific basis, but it still represented the average region of the cam lesion for our population. Clinically, the discretion of the surgeon is required to identify the areal extent based on subject-specific morphologic features. Second, although we have been implementing this technique in our surgical practice, we have yet to quantify the accuracy of resection in terms of removing only cortical bone, and we have yet to determine how resections based on this guideline affect fracture strength, kinematic function, and patient-reported outcomes. Third, the study populations included subjects who were screened radiographically based on $\alpha$ angles. We chose to use the frog-leg view because it has been shown to capture lesions in patients with cam FAI [12], but we acknowledge that it is possible that control subjects could have had cam lesions visible on other views. Fourth, the cam population was predominantly male and therefore we advocate for caution when using the cortical-cancellous boundary as a resection guideline when treating female patients. However, our previous analysis of cortical thickness found no differences in cortical thickness between male and female control subjects, suggesting that this guideline may be applicable for both sexes [1]. Fifth, the clinical history of cadaver femurs used as controls was not available; it is possible these individuals had hip pain. However, we excluded all femurs with evidence of cam FAI. Finally, it is unclear if normalization of anatomy defines the ideal resection for patients with cam FAI; slight overcorrection may be preferred by surgeons to reduce the likelihood of impingement.

Previous studies have evaluated the shape of the cam lesion and cortical thickness in patients with cam FAI
$[1,11,13,15]$. Our results agree with previous shape analyses of the outer cortex. Specifically, a previous SSM study found maximum deviation between mean cam and control shapes to be $3.3 \mathrm{~mm}$ in the anterolateral head-neck junction, which is in good agreement with our data [11]. Another study mapped the femoral head-neck offset in reference to the radius of the femoral head for cam FAI and control subjects; results showed that the lateral and anterior quadrants were larger for the patients when compared with the control subjects, similar to our findings [15]. Although not quantitatively comparable as a result of normalization to the diameter of the femoral head, the group differences found herein are qualitatively comparable. Harris et al. [13] fit the femoral head of patients with cam FAI to idealized shapes (spheres and conchoids) and found maximum deviations from a sphere of (mean $\pm \mathrm{SD}$ ) $5.0 \pm 0.4 \mathrm{~mm}$ for patients and $2.4 \pm 0.3 \mathrm{~mm}$ for control subjects and from a conchoid of $4.1 \pm 0.4 \mathrm{~mm}$ for patients and $1.8 \pm 0.3 \mathrm{~mm}$ for control subjects. Our measurements of maximum deviation of the control subjects and patients with cam FAI from the mean shape were slightly less than data reported by Harris et al. [13]. We suspect this is because normal femora are not spherical, and thus, deviations to an idealized geometry would be expected to be higher.

Removal of the subchondral cortical bone tended to yield a 3-D shape that underresected the cam lesion superiorly and overresected anteriorly. These errors can be partially attributed to our definition of a single region of resection which was superimposed on each subject femur, as any errors in identifying the proper region of resection may result in under- or overresection. Superiorly, subchondral cortical bone may have diminutive thickness compared with the anterior region of the femoral head, and thus removal of cortical bone over a standardized region based on a 1-mm threshold may not normalize femoral head anatomy given the variability in location of the cam lesion [28]. Collectively, these regional results indicate that when using the cortical-cancellous bone boundary as a surgical guideline, it is important not only to evaluate the specific 3-D morphologic features using intraoperative techniques (ie, full ROM arthroscopic views and fluoroscopic recreations of radiographic views) [4, 16, 17, 27], but also to consider the effects of varied cortical thickness when resecting the cam lesion. Still, the amount of underresection superiorly and overresection anteriorly was on the submillimeter level, which may be an improvement on current methods given that computer navigation methods have an accuracy of $\pm 1.9 \mathrm{~mm}$ and have been shown to be more precise than freehand techniques $[6,20]$. Additionally, observation of the cortical-cancellous boundary intraoperatively provides for real-time, subject-specific feedback on resection depth without requiring additional imaging or preoperative planning. 
Overall, simulated resection of subchondral cortical bone provided an effective method to determine cam resection depth for the population of cam femurs evaluated herein. In particular, resection of subchondral cortical bone to cortical-cancellous boundary yielded mean femoral anatomy for patients with cam FAI that was within a millimeter of the mean shape of control subjects without FAI with no differences in anatomic shape over the region of resection, with the numbers available. Nevertheless, differences in overall shape (ie, sphericity of the femoral head and shape of the greater trochanter) were still present after resection, indicating that removal of subchondral cortical bone over the cam lesion in patients with cam FAI alone cannot restore the shape of the entire proximal femur. The primary advantage of the proposed guideline is that the resection depth can likely be verified by inspection of arthroscopic images intraoperatively and thus does not require advanced imaging or 3-D modeling to generate a surgical plan. Future studies should establish the accuracy of the surgeon's resection in following this guideline and should determine if normalization of anatomy through resection of subchondral cortical bone alone improves clinical outcomes after hip arthroscopy.

Acknowledgments We thank Shireen Elhabian $\mathrm{PhD}$ and Praful Agrawal MS (Scientific Computing and Imaging Institute, University of Utah) for assistance with software development, and Trevor Hafer BS and Tyler Skinner BS (Department of Bioengineering, University of Utah) for assistance with image segmentation.

\section{References}

1. Atkins PR, Elhabian SY, Agrawal P, Harris MD, Whitaker RT, Weiss JA, Peters CL, Anderson AE. Quantitative comparison of cortical bone thickness using correspondence-based shape modeling in patients with cam femoroacetabular impingement. $J$ Orthop Res. 2016 Oct 27. [Epub ahead of print] doi:10.1002/jor. 23468.

2. Bogunovic L, Gottlieb M, Pashos G, Baca G, Clohisy JC. Why do hip arthroscopy procedures fail? Clin Orthop Relat Res. 2013;471:2523-2529.

3. Chan EF, Farnsworth CL, Koziol JA, Hosalkar HS, Sah RL. Statistical shape modeling of proximal femoral shape deformities in Legg-Calvé-Perthes disease and slipped capital femoral epiphysis. Osteoarthritis Cartilage. 2013;21:443-449.

4. Chow RM, Kuzma SA, Krych AJ, Levy BA. Arthroscopic femoral neck osteoplasty in the treatment of femoroacetabular impingement. Arthrosc Tech. 2014;3:e21-e25.

5. Clohisy JC, Nunley RM, Otto RJ, Schoenecker PL. The frog-leg lateral radiograph accurately visualized hip cam impingement abnormalities. Clin Orthop Relat Res. 2007;462:115-121.

6. Ecker TM, Puls M, Steppacher SD, Bastian JD, Keel MJ, Siebenrock KA, Tannast M. Computer-assisted femoral headneck osteochondroplasty using a surgical milling device an in vitro accuracy study. $J$ Arthroplasty. 2012;27:310-316.

7. Finner H. On a monotonicity problem in step-down multiple test procedures. J Am Stat Assoc. 1993;88:920-923.
8. Franklin SB, Gibson DJ, Robertson PA, Pohlmann JT, Fralish JS. Parallel analysis: a method for determining significant principal components. J Veg Sci. 1995;6:99-106.

9. Guevara-Alvarez A, Lash N, Beck M. Femoral head-neck junction reconstruction, after iatrogenic bone resection. J Hip Preserv Surg. 2015;2:190-193.

10. Harris JD, McCormick FM, Abrams GD, Gupta AK, Ellis TJ, Bach BR Jr, Bush-Joseph CA, Nho SJ. Complications and reoperations during and after hip arthroscopy: a systematic review of 92 studies and more than 6,000 patients. Arthroscopy. 2013;29:589-595.

11. Harris MD, Datar M, Whitaker RT, Jurrus ER, Peters CL, Anderson AE. Statistical shape modeling of cam femoroacetabular impingement. J Orthop Res. 2013;31:1620-1626.

12. Harris MD, Kapron AL, Peters CL, Anderson AE. Correlations between the alpha angle and femoral head asphericity: implications and recommendations for the diagnosis of cam femoroacetabular impingement. Eur J Radiol. 2014;83:788-796.

13. Harris MD, Reese SP, Peters CL, Weiss JA, Anderson AE. Threedimensional quantification of femoral head shape in controls and patients with cam-type femoroacetabular impingement. Ann Biomed Eng. 2013;41:1162-1171.

14. Hotelling H. The generalization of Student's ratio. In Kotz S, Johnson NL, eds. Breakthroughs in Statistics:Foundations and Basic Theory. New York, NY:Springer Science + Business Media; 1992:54-65.

15. Ito K, Minka MA 2nd, Leunig M, Werlen S, Ganz R. Femoroacetabular impingement and the cam-effect: a MRI-based quantitative anatomical study of the femoral head-neck offset. $J$ Bone Joint Surg Br. 2001;83:171-176.

16. Jackson TJ, Stake CE, Trenga AP, Morgan J, Domb BG. Arthroscopic technique for treatment of femoroacetabular impingement. Arthrosc Tech. 2013;2:e55-e59.

17. Larson CM, Wulf CA. Intraoperative fluoroscopy for evaluation of bony resection during arthroscopic management of femoroacetabular impingement in the supine position. Arthroscopy. 2009;25:1183-1192.

18. Maquer G, Burki A, Nuss K, Zysset PK, Tannast M. Head-neck osteoplasty has minor effect on the strength of an ovine cam-FAI model: in vitro and finite element analyses. Clin Orthop Relat Res. 2016;474:2633-2640.

19. Mardones RM, Gonzalez C, Chen Q, Zobitz M, Kaufman KR, Trousdale RT. Surgical treatment of femoroacetabular impingement: evaluation of the effect of the size of the resection. $J$ Bone Joint Surg Am. 2005;87:273-279.

20. Park CN, Nawabi DH, Christopher J, Conditt MA, Ranawat AS. Robotic-assisted femoral osteochondroplasty is more precise than a freehand technique in a Sawbone model. J Hip Preserv Surg. 2015;2:136-144.

21. Philippon MJ, Briggs KK, Yen Y-M, Kuppersmith DA. Outcomes following hip arthroscopy for femoroacetabular impingement with associated chondrolabral dysfunction: minimum two-year follow-up. J Bone Joint Surg Br. 2009;91:16-23.

22. R CoreTeam. R: A language and environment for statistical computing. $R$ Foundation for Statistical Computing. Available at: https://cran.r-project.org/doc/manuals/r-release/fullrefman.pdf. Accessed March 9, 2017.

23. Ross JR, Larson CM, Adeoyo O, Kelly BT, Bedi A. Residual deformity is the most common reason for revision hip arthroscopy: a three-dimensional CT study. Clin Orthop Relat Res. 2015;473:1388-1395.

24. Rothenfluh E, Zingg P, Dora C, Snedeker JG, Favre P. Influence of resection geometry on fracture risk in the treatment of femoroacetabular impingement: a finite element study. Am J Sports Med. 2012;40:2002-2008. 
25. Sardana V, Philippon MJ, de Sa D, Bedi A, Ye L, Simunovic N, Ayeni OR. Revision hip arthroscopy indications and outcomes: a systematic review. Arthroscopy. 2015;31:2047-2055.

26. Speirs AD, Beaule PE, Rakhra KS, Schweitzer ME, Frei H. Bone density is higher in cam-type femoroacetabular impingement deformities compared to normal subchondral bone. Osteoarthritis Cartilage. 2013;21:1068-073.

27. Stähelin L, Stähelin T, Jolles BM, Herzog RF. Arthroscopic offset restoration in femoroacetabular cam impingement: accuracy and early clinical outcome. Arthroscopy. 2008;24:5157.e1.
28. Treece GM, Gee AH, Mayhew PM, Poole KE. High resolution cortical bone thickness measurement from clinical CT data. Med Image Anal. 2010;14:276-290.

29. Wijdicks CA, Balldin BC, Jansson KS, Stull JD, LaPrade RF, Philippon MJ. Cam lesion femoral osteoplasty: in vitro biomechanical evaluation of iatrogenic femoral cortical notching and risk of neck fracture. Arthroscopy. 2013;29:1608-1614.

30. Zingg PO, Buehler TC, Poutawera VR, Alireza A, Dora C. Femoral neck fractures after arthroscopic femoral neck osteochondroplasty for femoroacetabular impingement. Knee Surg Sports Traumatol Arthrosc. 2014;22:926-931. 